Revista Brasileira de Agricultura Irrigada v.9, nº.4, p. 232 - 238, 2015

ISSN 1982-7679 (On-line)

Fortaleza, CE, INOVAGRI - http://www.inovagri.org.br

DOI: $10.7127 /$ rbai.v9n400307

Protocolo 307.15 - 25/05/2015 Aprovado em 08/07/2015

\title{
ESTACIONALIDADE DA EVAPOTRANSPIRAÇ̃̃O E PRODUTIVIDADE DA ÁGUA NA MICROBACIA DO CÓRREGO DA MULA EM SANTA FÉ DO SUL - SP
}

\author{
Daniel Coaguila ${ }^{1}$, Fernando Braz Tangerino Hernandez ${ }^{2}$, Antônio Heriberto de Castro \\ Teixeira $^{3}$
}

\section{RESUMO}

A microbacia do córrego da Mula se apresenta como um sócio-ecossistema sensível, com talvegues assoreados, ausência de matas ciliares e nascentes desprotegidas. A capacidade de gerar e armazenar água pela microbacia é excedida pela demanda em períodos de seca, agravando-se em secas prolongadas. Sendo assim necessário avaliar o comportamento da água acima do solo na microbacia, realizado nas épocas seca e úmida dos anos 2013 e 2014. Foram adquiridas imagens do Landsat-8 (OLI e TIRS) para os meses com menor e maior precipitação dos dois anos. Com o modelo Simple Algorithm for Evapotranspiration Retrieving (SAFER) foram calculadas a evapotranspiração atual (ET), biomassa (BIO) e produtividade da agua (WP), apresentando valores médios na microbacia de $0,39 \mathrm{~mm} \mathrm{dia}{ }^{-1}$, $9,17 \mathrm{~kg} \mathrm{ha}^{-1} \mathrm{dia}^{-1}$ e $1,73 \mathrm{~kg} \mathrm{~m}^{-3}$ respectivamente, para o período seco do ano $2013 \mathrm{e} \mathrm{de} 0,40$ $\mathrm{mm} \mathrm{dia}{ }^{-1}, 10,98 \mathrm{~kg} \mathrm{ha}^{-1} \mathrm{dia}^{-1}$ e $1,92 \mathrm{~kg} \mathrm{~m}^{-3}$ no período seco do ano 2014 . No período úmido em 2014 os valores médios de ET, BIO e WP foram de 1,35 mm dia ${ }^{-1}, 52,19 \mathrm{~kg} \mathrm{ha}^{-1} \mathrm{dia}^{-1} \mathrm{e}$ $3,39 \mathrm{~kg} \mathrm{~m}^{-3}$, respectivamente. No ano 2014 pela restrição hídrica no período seco inerente à época, a ET representa o $29,63 \%$ da capacidade evapotranspirativa quando comparado com a época úmida do mesmo ano. Da mesma forma a BIO representa o 21,04\% do potencial da microbacia e uma WP com 57,23\% de eficiência na época seca. A microbacia do córrego da Mula, em época seca, opera à metade da sua capacidade conhecida para o ano 2014.

Palavras-chave: biomassa, Landsat-8, SAFER

\section{SEASONALITY OF EVAPOTRANSPIRATION AND WATER PRODUCTIVITY IN THE MICROWATERSHED OF MULA STREAM, SANTA FÉ DO SUL - SP}

\footnotetext{
ABSTRACT

${ }^{1}$ Mestre, Doutorando em Agronomia, Área de Hidráulica e Irrigação, UNESP, Ilha Solteira - SP, Brasil, Rua Monção 226, CEP: 15385-000, e-mail: tuheraldo@gmail.com

${ }^{2}$ Eng. Agrônomo, Prof. Doutor, Área de Hidráulica e Irrigação, UNESP, Ilha Solteira - SP, Brasil, Rua Monção 226, CEP: 15385-000, e-mail: fbthtang@agr.feis.unesp.br

${ }^{3}$ Doutor, Pesquisador, EMBRAPA Monitoramento por Satélite, Campinas - SP, Brasil, Av. Soldado Passarinho 303 - Fazenda Chapadão, CEP: 13070-115, e-mail: heriberto.teixeira@embrapa.br
} 
The microwatershed of Mula stream is presented as a sensitive socio-ecosystem, with thalwegs sedimented, absence of riparian forests and unprotected springs. The ability to generate and store water in the microwatershed is exceeded by demand in periods of drought, worsening during periods of prolonged droughts. Being necessary to evaluate the behavior of above-ground water in the microwatershed, conducted in the dry and wet seasons of 2013 and 2014. Were acquired imagery set of Landsat-8 (OLI and TIRS) for the months with the lowest and highest precipitation of 2013 and 2014. Using the Simple Algorithm for Retrieving Evapotranspiration model (SAFER) were calculated actual evapotranspiration (ET), biomass (BIO) and water productivity (WP) showing average values in the microwatershed of 0.39 mm day ${ }^{-1}, 9.17 \mathrm{~kg} \mathrm{ha}^{-1}$ day $^{-1} \mathrm{e} 1.73 \mathrm{~kg} \mathrm{~m}^{-3}$, respectively, in the dry season of 2013 and 0.40 $\mathrm{mm}$ day $^{-1}, 10.98 \mathrm{~kg} \mathrm{ha}^{-1}$ day $^{-1}$ e $1.94 \mathrm{~kg} \mathrm{~m}^{-3}$ in the dry season of 2014. During the wet season of 2014 mean values of ET, BIO and WP were $1.35 \mathrm{~mm} \mathrm{day}^{-1}, 52.19 \mathrm{~kg} \mathrm{ha}^{-1}$ day $^{-1}$ e $3.39 \mathrm{~kg}$ $\mathrm{m}^{-3}$, respectively. In 2014 the water restriction in the dry season inherent to the period, the ET represents $29.63 \%$ on the evapotranspiration capacity when compared to the wet season of the same year. Similarly, BIO represents $21.04 \%$ of the potential of the microwatershed and a WP with $57.23 \%$ efficiency in the dry season. The microwatershed of Mula stream, in dry season, works at half of its capacity known for 2014.

Keywords: biomass, Landsat-8, SAFER

\section{INTRODUÇÃO}

A água é diferente de todos os outros recursos naturais, devido à suas propriedades físicas, importância cultural e religiosa, complexas características econômicas e pelo seu papel essencial para o apoio na vida do planeta (Grover, 2006). Sendo a água superficial o principal recurso hídrico renovável acessível para usos humanos. Numa bacia hidrográfica, o escoamento superficial é aproximadamente o equilíbrio entre a precipitação recebida da atmosfera e a evapotranspiração (ET).

A produção agropecuária em todos os tipos de ambientes tem como fator limitante a disponibilidade de água, que trará um impacto maior nos próximos anos se forem confirmadas as previsões de mudança climática global realizadas por organizações internacionais, como a do Intergovernmental Panel on Climate Change (IPCC), onde é relatado o incremento nas emissões de $\mathrm{CO}_{2}$ e são modelados cenários futuros com consequências impactantes se os governantes não adotarem providências para mitigar estes efeitos sobre o nosso planeta (IPCC, 2014).

A região noroeste do Estado de São Paulo caracteriza-se como uma área tipicamente agrícola com períodos sucessivos de déficits hídricos, o que faz com que o uso eficiente da água seja necessário para assegurar a produtividade (Hernandez et al., 2003; Santos et al., 2010).

No município de Santa Fé do Sul (região noroeste do Estado de São Paulo), particularmente na microbacia do córrego da Mula, a água, além de ser requerida para o uso da população, é requerida também pela agropecuária. Este cenário sensível vem sendo crítico em estiagens prolongadas, comprometendo a agropecuária, o abastecimento de agua potável da cidade e à saúde pública, como se observou em 2014, quando o racionamento da água foi imposto à população no segundo semestre, e mais recentemente em abril e maio do presente ano onde uma água "escura" tem saído das torneiras dos moradores do Município de Santa Fé do Sul.

Em microbacias hidrográficas frágeis na oferta e uso conflitivo da água, como o caso da microbacia do córrego da Mula, é de extrema importância que sejam realizadas avaliações técnicas que possam gerar informações que irão contribuir para o uso adequado da água. Estes estudos podem ser realizados através da determinação da evapotranspiração, da biomassa e da produtividade da água da bacia. Estes parâmetros podem ser determinados com precisão 
a partir de métodos indiretos, os quais estão baseados em medidas radiométricas obtidas a partir de sensores remotos (Allen et al., 2007, Bezerra et al., 2008, Li et al., 2008).

Desta forma, tendo em vista o cenário de fragilidade hídrica e a carência de informações técnicas que possam subsidiar o planejamento do uso adequado da água, este trabalho teve por objetivo estimar a evapotranspiração, a biomassa e a produtividade da água da microbacia do córrego da Mula através do sensoriamento remoto.

\section{MATERIAL E MÉTODOS}

A área de estudo localiza-se na microbacia do córrego da Mula, município de Santa Fé do Sul - SP (Figura 1), com 370 m de altitude média, com uma área de 4.342 hectares, $34 \mathrm{~km}$ de perímetro e uma hidrografia de $38,88 \mathrm{~km}$. O uso do solo da microbacia é heterogênea em toda sua extensão, estando presentes culturas perenes (citros e seringueira), pastagem, espécies invasoras (Typha sp. em sua grande maioria) ao longo do córrego, poucos fragmentos de mata remanescente e quase todas as nascentes desprotegidas.

O clima da região, segundo classificação de Köppen, é do tipo Aw, definido como tropical úmido com estação chuvosa no verão e seca no inverno, temperatura média anual de $24,5{ }^{\circ} \mathrm{C}$ e precipitação pluvial média anual de $1.232 \mathrm{~mm}$, com umidade média anual de $64,8 \%$ (Hernandez et al., 1995). O solo pertence à classe taxonômica Argissolo Vermelho Amarelo Eutrófico (Oliveira et al., 1999).

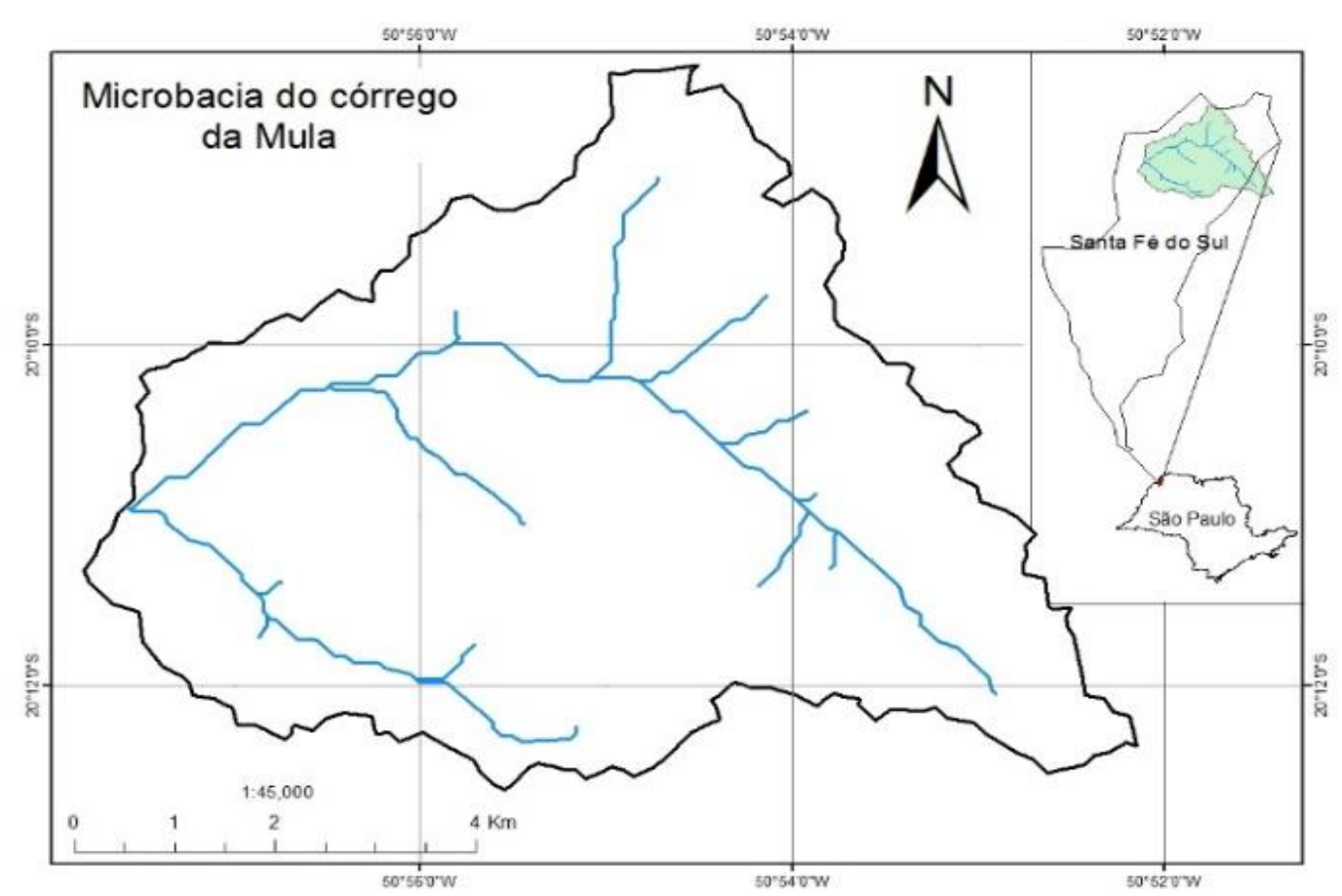

Figura 1. Área de estudo, microbacia do córrego da Mula, Santa Fé do Sul - SP.

As imagens utilizadas foram adquiridas do Landsat 8 (OLI e TIRS) com orbita 222 e ponto 74 através do U.S. Geological Survey (www.usgs.gov), dos anos 2013 (doy: 249) e 2014 (doy: 76 e 252) correspondendo a estação seca e úmida. Já as variáveis agrometeorológicas foram adquiridas por meio da Rede Agrometeorológica do Noroeste Paulista, constituída por oito estações beneficiando uma área de aproximadamente 7.465 $\mathrm{km}^{2}$ (http://clima.feis.unesp.br).

Antes da estimativa da evapotranspiração, biomassa e produtividade da água via sensoriamento remoto, as imagens foram processadas com correções geométricas, calibrações radiométricas, calibrações atmosféricas e informações biofísicas. $\mathrm{O}$ processamento das 
imagens do satélite foi realizado segundo a metodologia do U.S. Geological Survey (www.usgs.gov) para as calibrações radiométricas e atmosféricas, foram utilizadas as metodologias de Teixeira (2010), Teixeira et al. (2012), Teixeira et al. (2013) e Hernandez et al. (2014) para a modelagem da evapotranspiração atual pelo modelo Simple Algorithm for Retrieving
Evapotranspiration (SAFER), cálculo da biomassa e produtividade da água (Figura 2).

Para o geoprocessamento das informações foi utilizado o software ArcGIS ${ }^{\circledR} \quad 10.0$ $\left(\right.$ ArcMap $\left.^{\mathrm{TM}}\right)$ do ESRI que na função model builder possibilitou os cálculos dos dados no formato matricial (raster).

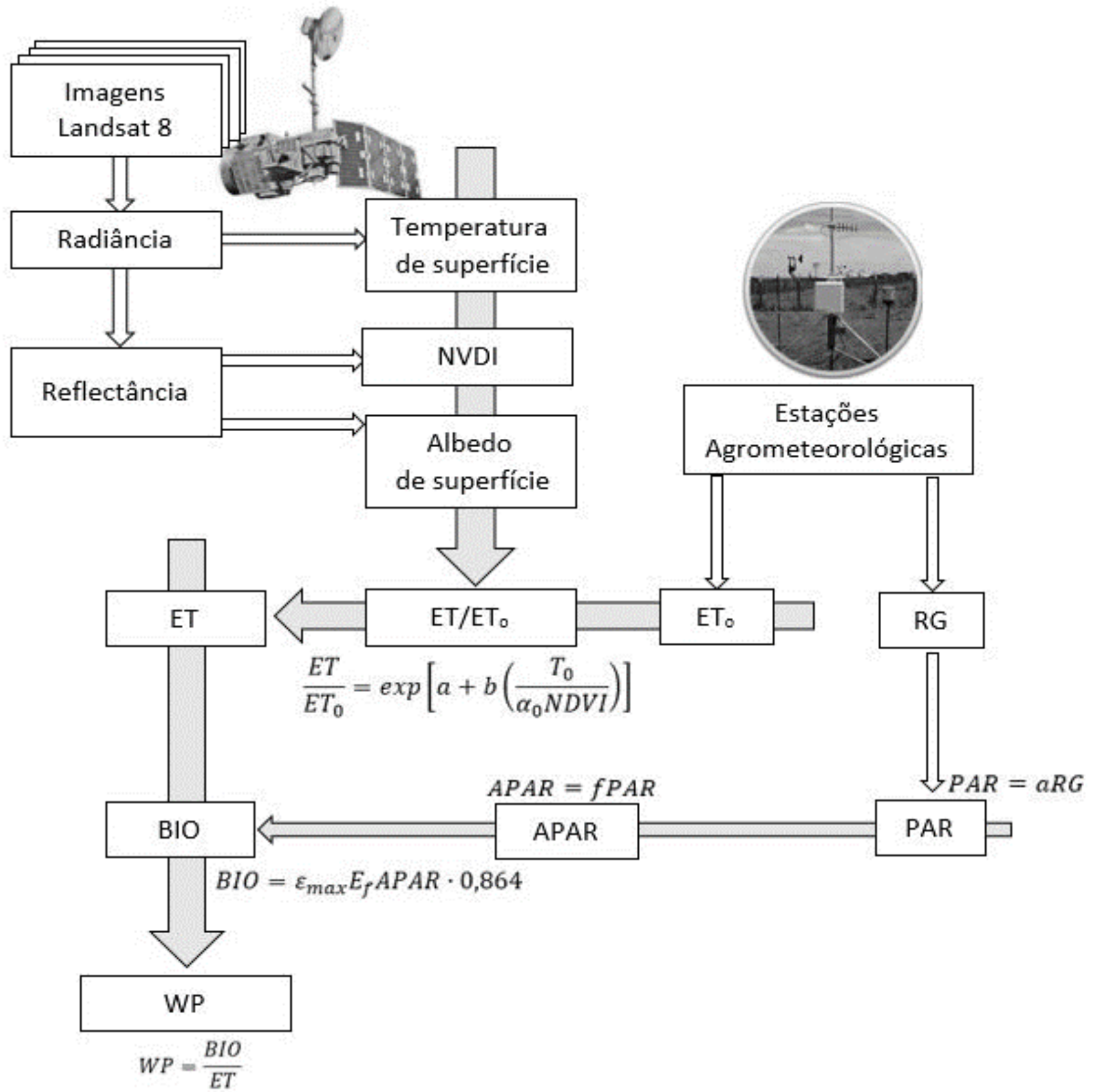

Figura 2. Fluxograma para o cálculo da evapotranspiração, biomassa e produtividade da água a partir do modelo Simple Algorithm for Retrieving Evapotranspiration (SAFER). 


\section{RESULTADOS E DISCUSSÃO}

Os valores de evapotranspiração na época seca (setembro) do ano 2013 teve seus valores consideravelmente baixos e altamente heterogêneos (Tabela1) devido à falta de chuvas superiores a $10 \mathrm{~mm}$ na região durante 71 dias (http://clima.feis.unesp.br), resultados próximos aos encontrados foram apresentados por Franco et al. (2014) e Coaguila et al. (2015). Valores $102,56 \%$ superiores de evapotranspiração foram calculados para a época seca do ano 2014, quando comparado com a mesma época do ano 2013
(Tabela 1), já que nesse ano a região teve apenas 44 dias sem chuvas superiores a $10 \mathrm{~mm}$, o que significa que no ano 2014 (época seca, 09 setembro) a microbacia esteve exposta a 1,6 vezes a mais chuva quando comparado com a época seca do ano 2013 (05 setembro).

$\mathrm{Na}$ época úmida do ano 2014 (17 março) a evapotranspiração da microbacia do córrego da Mula foi de 1,35 $\mathrm{mm} \mathrm{dia}^{-1}$ (Tabela 1, Figura 3a), típica da época pela presença de chuvas $(236,8$ mm acumulado no mês de março até a data de aquisição da imagem).

Tabela 1. Média $(\bar{x} \pm \mathrm{DP})$ de Evapotranspiração (ET), biomassa (BIO) e produtividade da água (WP) da microbacia do córrego da Mula.

\begin{tabular}{|c|c|c|c|c|c|}
\hline Ano & Época & DOY & $\begin{array}{c}\text { ET } \\
\left(\mathrm{mm} \mathrm{dia}^{-1}\right)\end{array}$ & $\begin{array}{c}\text { BIO } \\
\left(\mathrm{kg} \mathrm{ha}^{-1} \mathrm{dia}^{-1}\right)\end{array}$ & $\begin{array}{c}\text { WP } \\
\left(\mathrm{kg} \mathrm{m}^{-3}\right)\end{array}$ \\
\hline 2013 & Seca & 249 & $0,39 \pm 0,45$ & $9,17 \pm 14,58$ & $1,73 \pm 0,80$ \\
\hline \multirow{2}{*}{2014} & Úmida & 76 & $1,35 \pm 0,65$ & $52,19 \pm 30,45$ & $3,39 \pm 1,08$ \\
\hline & Seca & 252 & $0,40 \pm 0,45$ & $10,98 \pm 17,27$ & $1.94+0.89$ \\
\hline
\end{tabular}

A biomassa acumulada na microbacia em estudo para a época seca do ano 2013 foi de 9,17 $\mathrm{kg} \mathrm{ha}^{-1} \mathrm{dia}^{-1}$ inferior à acumulada na mesma época do ano $2014\left(10,98 \mathrm{~kg} \mathrm{ha}^{-1} \mathrm{dia}^{-1}\right.$, Tabela 1). A biomassa depende da evapotranspiração que foi inferior no ano 2013 se comparada com o ano 2014. Resultados inferiores $\left(3,86 \mathrm{~kg} \mathrm{ha}^{-1} \mathrm{dia}^{-1}\right)$ foram apresentados para a bacia do córrego Cabeceira Comprida (Coaguila et al., 2015) em setembro de 2011 utilizando imagens do Landsat5. Já na época úmida do ano 2014 a microbacia apresentou uma média de $52,19 \mathrm{~kg} \mathrm{ha}^{-1} \mathrm{dia}^{-1}$, valor que acompanha a evapotranspiração $\left(1,35 \mathrm{~mm}\right.$ dia $^{-}$ $\left.{ }^{1}\right)$ da data em estudo. Observa-se uma alta heterogeneidade da biomassa na época úmida o que não acontece na época seca pelo longo período de estiagem a qual esteve submetida a área de estudo (Figura 3b). Pode acontecer também que o estresse relacionado com o clima, resulte em menor absorção de carbono e biomassa vegetal sobre a estação de crescimento, reduzindo assim a produção de culturas anuais e o rendimento (Marshall et al., 2012).
Sabendo-se que a produtividade da água é a razão da biomassa pela evapotranspiração, esperase que a eficiência média da bacia $\left(\mathrm{kg} \mathrm{m}^{-3}\right)$ seja inferior na época seca quando comparada com a época úmida. Os resultados mostram que durante a época seca nos anos 2013 e 2014 a microbacia em estudo em média produziu 1,73 e $1,94 \mathrm{~kg} \mathrm{~m}^{-3}$ respectivamente (Tabela 1). Coaguila et al. (2015) para a época seca do 2011 obteve valores de 1,13 $\mathrm{kg} \mathrm{m}^{-3}$ para a microbacia do córrego Cabeceira Comprida, com $3,4 \mathrm{~kg} \mathrm{~m}^{-3}$ para a mata remanescente, $3,9 \mathrm{~kg} \mathrm{~m}^{-3}$ para as culturas perenes e $2,7 \mathrm{~kg} \mathrm{~m}^{-3}$ para as espécies invasoras, por sua vez Franco et al. (2014) descreveu produtividades na vegetação de $2,3-3,4 \mathrm{~kg} \mathrm{~m}^{-3}$ na região do noroeste Paulista.

$\mathrm{Na}$ época úmida na microbacia em estudo (Figura 3c) a produtividade da água em média foi de $3,35 \mathrm{~kg} \mathrm{~m}^{-3}$ o que corrobora os dados obtidos por Franco et al. (2014) que descreveu produtividades médias de $2,3-3,4 \mathrm{~kg} \mathrm{~m}^{-3}$ no noroeste paulista. 

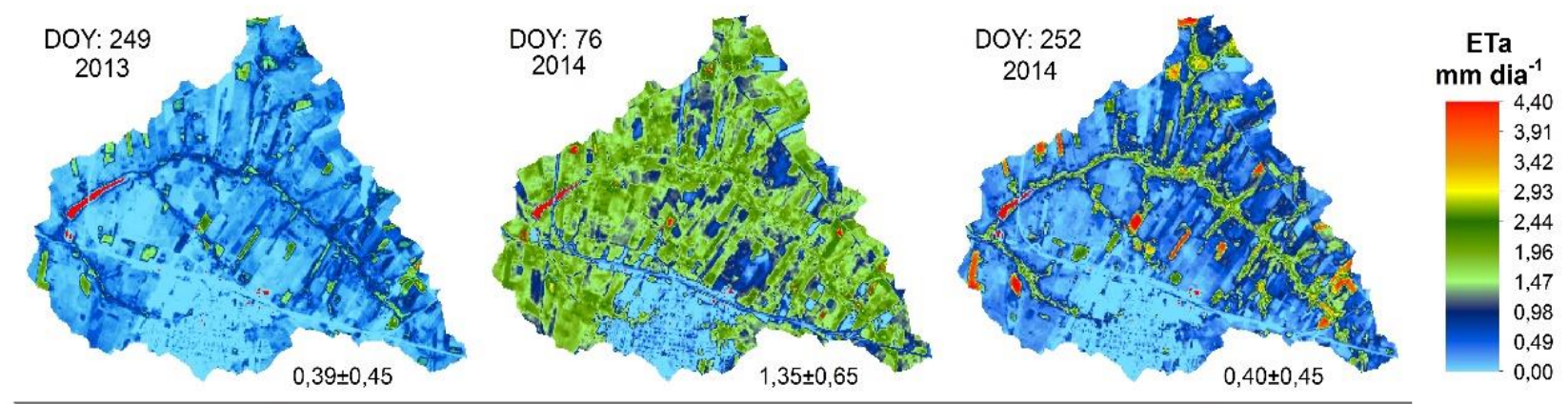

(a)
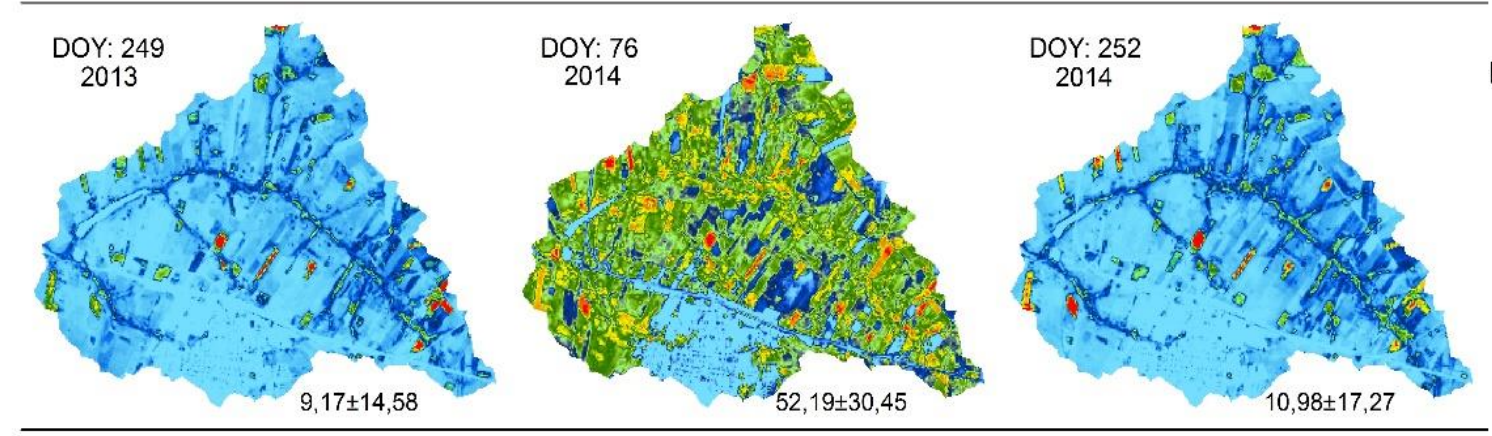

BIO

$\mathrm{kg} \mathrm{ha}^{-1} \mathrm{dia}^{-1}$

(b)
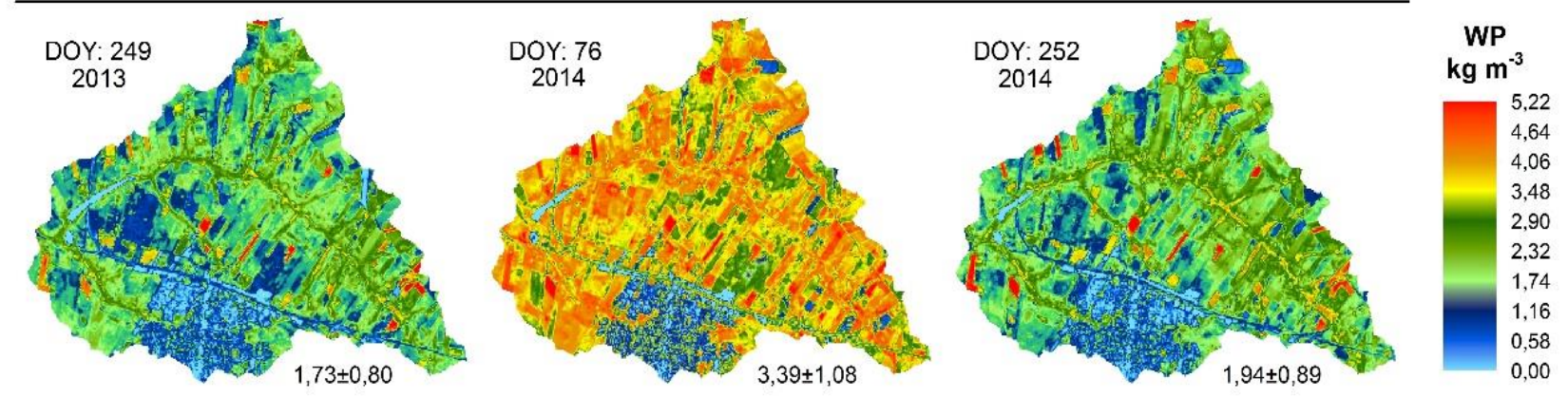

(c)

Figura 3. (a) Evapotranspiração (ET), (b) Biomassa (BIO) e (c) Produtividade da água (WP) nos períodos seco (setembro 2013 e 2014, doy 249 e 252 respectivamente) e úmido (março 2014, doy 76) da microbacia do córrego da Mula. $(\bar{x} \pm D P)$.

\section{CONCLUSÕES}

Os parâmetros de evapotranspiração, biomassa e produtividade da água da microbacia do córrego da Mula foram devidamente determinados através das metodologias propostas. Os parâmetros obtidos na época seca foram inferiores aos valores obtidos na época úmida nos anos 2013 e 2014. Os valores calculados para a época seca dos anos 2013 e 2014 foram diferentes em função da estiagem prolongada durante o ano 2013. A alta heterogeneidade espacial da evapotranspiração, biomassa e produtividade da água deve-se principalmente à ausência de mata ciliar e ás nascentes desprotegidas.

\section{AGRADECIMENTOS}

Os autores agradecem ao Programa de Apoio a Estudantes de Doutorado do Exterior (PAEDEx) da Universidade Estadual Paulista (UNESP), à Associação Universitária Iberoamericana de Pós-graduação (AUIP) processo 2012, que concederam a bolsa de estudo de doutorado ao primeiro autor.

\section{REFERÊNCIAS BIBLIOGRÁFICAS}

ALLEN, R.G. et al. Satellite-based energy balance for mapping evapotranspiration with internalized calibration (METRIC) - Applications. Journal of Irrigation and Drainage Engineering, v. 133, n. 4, 
p. 395-405, 2007.

BEZERRA, B.G.; SILVA, B.B. da; FERREIRA, N.J. Estimativa da evapotranspiração real diária utilizando-se imagens digitais TM - Landsat 5. Revista Brasileira de Meteorologia, v. 23, n. 3, p. 305-317, 2008.

COAGUILA, D.; HERNANDEZ, F.B.T.; TEIXIERA, A.H.C. Eficiência no uso da água da vegetação na microbacia hidrográfica do córrego Cabeceira Comprida. In: XVII Simpósio Brasileiro de Sensoriamento Remoto, 2015, João Pessoa. Anais... Anais do XVII SBSR, 3044 - 3051.

FRANCO, R.M.; HERNANDEZ, F.B.T.; TEIXEIRA, A.H.C. Water productivity of different land uses in watersheds assessed from satellite imagery Landsat 5 Thematic Mapper. In: SPIE Remote Sensing Symposium, 2014, Amsterdam. Anais... Proceedings of Spie, v. 9239-86.

GROVER, V. I. General Overview. In:

Water global common and global problems. USA: Science Publisher, 2006. p. 3-16.

HERNANDEZ, F.B.T.; de SOUZA, S.A.V.; ZOCOLER, J.L.; FRIZZONE, J.A. Simulação e efeito de veranicos em culturas desenvolvidas na região de Palmeira d'Oeste, Estado de São Paulo. Engenharia Agrícola, v. 23, p. 21-30, 2003.

HERNANDEZ, F.B.T.; LEMOS Filho, M.A.F.; BUZETTI, S. Software HIDRISA e o Balanço Hídrico de Ilha Solteira. UNESP/FEIS/Área de Hidráulica e Irrigação, Ilha Solteira, Série Irrigação, n. 1, 45 p., 1995.

HERNANDEZ, F.B.T.; NEALE, C.M.U.; TEIXEIRA, A.H.; Taghvaeian, S. Determining large scale actual evapotranspiration using agrometeorological and remote sensing data in the northwest of Sao Paulo State, Brazil. Acta Horticulturae, v. 1038, p. 263-270, 2014.

Intergovernmental Panel on Climate Change - IPCC. Climate Change 2014 Synthesis Report. Copenhague, 2014. 114p.

LI, H. ; ZHENG, L.; LEI, Y.; LI, C.; LIU, Z.;
ZHANG, S. Estimation of water consumption and crop productivity of winter wheat in North China Plain using remote sensing technology. Agricultural Water Management, v. 95, n. 11, p. 1271-1278, 2008.

MARSHALL, M.T.; FUNK, C.; MICHAELSEN, J. Agricultural Drought Monitoring in Kenya Using Evapotranspiration Derived from Remote Sensing and Reanalysis Data. In: WARDLOW, B.D.; ANDERSON, M.C.; VERDIN, J.P. Remote Sensing of Drought Innovative Monitoring Approaches. USA: CRC Press Inc., 2012. p. 169190.

OLIVEIRA, J.B.; CAMARGO, M.N.; ROSSI, M.; CALDERANO Filho, B. Mapa pedológico do Estado de São Paulo: legenda expandida. Campinas: Instituto Agronômico, 1999. 64p.

SANTOS, G.O.; HERNANDEZ, F.B.T.; ROSSETTI, J.C. Balanço hídrico como ferramenta ao planejamento agropecuário para a região de Marinópolis, noroeste do estado de São Paulo. Revista Brasileira de Agricultura Irrigada, v. 4, p. 142-149, 2010.

TEIXEIRA, A.H.C. Determining regional actual evapotranspiration of irrigated and natural vegetation in the São Francisco river basin (Brazil) using remote sensing an Penman-Monteith equation. Remote Sensing, v. 2, p. 1287-1319, 2010.

TEIXEIRA, A.H.C.; HERNANDEZ, F.B.T.; LOPES, H.L. Application of Landsat images for quantifying the energy balance under conditions of land use changes in the semi-arid region of Brazil. In: SPIE Remote Sensing Symposium, 2012, Edimburgo. Anais... Proceedings of Spie, v. 8531, p. 85310P-1-85310P-9.

TEIXEIRA, A.H.C.; HERNANDEZ, F.B.T.; LOPES, H.L.; SCHERER-WARREN, M.; BASSOI, L.H. A Comparative Study of Techniques for Modeling the Spatiotemporal Distribution of Heat and Moisture Fluxes in Different Agroecosystems in Brazil. In: Petropoulos, G.P. (Org.). Remote Sensing of Energy Fluxes and Soil Moisture Content. Florida: CRC Group, Taylor and Francis, 2013. p. 165-187. 